\title{
A REVIEW OF LENGTH-WEIGHT RELATIONSHIPS OF FRESHWATER FISHES IN MALAYSIA
}

\author{
Amonodin MOHAMAD RADHI *, Mohd-Fadzil NURUL FAZLINDA **, \\ Mohammad Noor Azmai AMAL *** and Hashim ROHASLINEY*
}

\begin{abstract}
* Department of Environmental Management, Faculty of Environmental Studies, Universiti Putra Serdang, Selangor, Malaysia, MY-43400,radhi_aminuddin@yahoo.com,rohasliney@upm.edu.my

** School of Biological Sciences, Universiti Sains Malaysia, USM, Pulau Pinang, Malaysia, MY-11800, linda_fadzil91@yahoo.com

*** Department of Biology, Faculty of Science, Universiti Putra Malaysia, Serdang, Selangor, Malaysia, MY-43400,mnamal@upm.edu.my
\end{abstract}

DOI: 10.1515/trser-2018-0005

KEYWORDS: Freshwater fishes, Length-weight relationships, Malaysia.

\section{ABSTRACT}

This manuscript reviews the length-weight relationships (LWRs) of freshwater fishes in Malaysia. A total of 102 LWRs of fishes gathered from literature pertaining to 64 freshwater fish species were analysed. A meta-analysis from 13 previous reports showed that the $b$ values was ranged from 2.19 (Clarias batrachus) to 4.106 (Barbodes binotatus). Out of 64 observed species, 47 species (11 families) experienced positive allometric growth, while another 23 species (eight families) and 31 species (12 families) were recorded under isometric and negative allometric growth, respectively. The fish LWRs observed can be used as an indicator of environmental changes and fish ecological health for freshwater fishes in Malaysia.

ZUSAMMENFASSUNG: Eine Überprüfung der Länge-Gewicht Beziehungen von Süßwasserfischen in Malaysia.

Vorliegende Arbeit befasst sich mit der Überprüfung der Längengewichtsverhältnisse (LWRs) von Süßwasserfischen in Malaysia. Insgesamt wurden 102 LWRs von 64 Süßwasserfischarten anhand von Literaturangaben analysiert. Eine Metaanalyse aus 13 früheren Berichten zeigte, dass die b-Werte von 2,19 (Clarias batrachus) bis 4.106 (Barbodes binotatus) reichen. Von 64 beobachteten Arten zeigten 47 Arten (elf Familien) ein positives allometrisches Wachstum, während weitere 23 Arten (acht Familien) und 31 Arten (12 Familien) unter isometrischem und negativem allometrischem Wachstum aufgezeichnet wurden. Die beobachteten Fische LWRs können als Indikatoren für Umweltveränderungen und Bewertung der ökologischen Gesundheit von Süßwasserfischen in Malaysia verwendet werden. Malaezia.

REZUMAT: O revizuire a relaţiilor lungime-greutate a peştilor de apă dulce din

Acestă lucrare analizează dinamica în timp a relațiilor lungime-greutate (LWRs) la specii de pești de apă dulce din Malaezia. Au fost analizate un total de 102 LWRs selectate din literatura de specialitate, referitoare la 64 de specii de pești de apă dulce. O meta-analiză a datelor din 13 rapoarte anterioare a arătat că valorile b a variat între 2,19 (Clarias batrachus) și 4,106 (Barbodes binotatus). Din 64 de specii observate, 47 de specii (11 familii) au cunoscut 0 creștere alometrică pozitivă, în timp ce alte 23 de specii (opt familii) și 31 specii (12 familii) au fost înregistrate în creștere alometrică izometrice și negative, respectiv. LWRs observat a peștilor poate fi folosit ca un indicator al schimbărilor de mediu și de sănătate ecologică pentru pești de apă dulce din Malaezia. 


\section{INTRODUCTION}

A total of 1,951 species of freshwater and marine fish belonging to 704 genera and 186 families have been recorded in Malaysia (Chonget al., 2010). In Peninsular Malaysia alone, about 278 native species were recorded by Lim and Tan (2002). The IUCN Red List of Threatened Species listed 1,275 fish species that are threatened from around the world and surprisingly, Malaysia hosts over 49 threatened fish species (Vié et al., 2008). At present, the indigenous species such as Jullien's golden carp (Probarbus jullieni), mahseer (Tor tambroides), Hoven's carp (Leptobarbus hoeveni), hampala barb (Hampala macrolepidota), climbing perch (Anabas testudineus), giant snakehead (Channa micropeltes), Asian arowana (Scleropages formosus), pangasiid catfishes (Pangasius nasutus, Pangasigodon waandersii), giant river catfish (Wallago leerii), catfish (Clarias macrocephalus) and giant gourami (Osphronemus goramy) have dwindled in great numbers continuously due to unsustainable fishing activities and could only be conserved probably in the inaccessible or remote areas of the country (Chew and Zulkafli, 2007). Moreover, freshwater ecosystems are demarcated due to the potential impacts of anthropogenic activities, giving them precedence for research, conservation, and sustainable management. Chong et al., (2010) reported that freshwater fishes recently encompassed the highest percentage of threatened fish species followed by estuarine among the aquatic systems. The diversity and distribution of freshwater fishes in Malaysia has been disrupted rapidly due to developmental pressure and modification of fish habitat. Future declines can therefore negatively affect freshwater biodiversity (Zakaria-Ismail, 1991; Bowen et al., 2003).

Length-weight analysis is a useful analysis in estimating average weight of fish caught from samples of lengths of fish caught (Hilborn and Walters, 2001; Adaka et al., 2015). The length-weight relationship (LWR) of fishes is important in fisheries and fish biology studies to provide information regarding growth patterns and the condition of fish species (Bagenal and Tesch, 1978). The study of LWRs is also important for the conservation and management of fishes in aquatic system, including freshwater system (Lawson, 2011), which are the most plausible area of efficacious pollutant sources due to the frequency, duration and magnitude of anthropogenic influences (Rahel, 2007; Francis, 2012).

Ricker (1975) has expressed the relationship between length $(L)$ and weight $(W)$ as W $=a \mathrm{~L}^{b}$. The constant value, $a$, has the ability to interpret body shape. For example, when the $a$ value is 0.001 , it shown that the fish is more eel-like, 0.008 more elongated, 0.013 more fusiform and 0.018 more short and deep (Froese, 2006). The value of $b$ exponent depicts very important information of fish growth capability to predict the health of the fish. When $b$ is equal to 3 , the increase in weight is isometric which means the fish length and weight increases proportionally (Santos et al., 2002). If the value $b>3$ (positive allometric), then there is a significant positive relationship between weight and fish length which indicates that weight will increase with increasing length, thus as the fish length increases the more rotund the fish will become; while when $\mathrm{b}<3$ (negative allometric), then the weight will decrease with increase of fish length, thus as fish length increase the less rotund the fish will become (Jones et al., 1999). Negative allometric growth pattern shows that there are possibilities of unsuitable environmental conditions which influence the condition to these species. Fish growth may be influenced by many biotic and abiotic factors such as phytoplankton abundance, predation, water temperature, and dissolve oxygen concentrations among others which may not favour the survival of all the species in the ecosystem (Atama et al., 2013). 
Currently, researchers also use Bayesian length weight to compare between genera. Bayesian methods combine existing knowledge (prior probabilities) with additional knowledge derived from new data (the likelihood function) (Froese et al., 2014). This results in updated knowledge (posterior probabilities), which can be used as priors in subsequent analyses and thus provide learning chains in science (Kurikka et al., 2014). This method uses FishBase (www.fishbase.org) as an online tool that facilitates the analysis of existing parameters and of new weight-at-length data (Froese et al., 2014). In FishBase, the Bayesian approach has been also used in the analysis of LWRs for estimating LWR for species for which this information is not available by using over 5,000 LWR records for over 1,800 species (Froese et al., 2014). In this review, we gathered 102 LWRs from the literature compromising of 64 freshwater fish species from Malaysian inland waters in order to determine their robustness and condition.

\section{MATERIAL AND METHODS}

All fish LWRs presented here are collected data of field studies conducted during 2000-2015 in freshwaters environment of Malaysia, and are consistent with the format suitable for inclusion in FishBase. The sources of these LWRs are gathered from various journals and technical reports. The majority of the original LWRs stated in this study were presented in $\mathrm{W}=a \mathrm{~L}^{b}$ equation form. But, there were several studies that provide $a$ and $b$ values only without the full equation. To standardize the review, the value was arranged in $\mathrm{W}=a \mathrm{~L}^{b}$ equation form. All reported $a$ and $b$ values are analysed to obtain the descriptive statistical analysis (mean, minimum and maximum values). Ten fish species that have three or more LWRs were chosen to be compared with Bayesian LWRs obtained from FishBase (Froese and Pauly, 2016).

\section{RESULTS AND DISCUSSION}

A total of 102 LWRs were gathered from the literatures, referring to 64 species and belonging to 20 families of Malaysian fresh water fishes (Tab. 1). The value of the slope of regression, $b$, in the plot of $\log \mathrm{W}$ against log $\mathrm{L}$ ranged from 2.190 for Clarias batrachus collected in Pahang, to 4.106 for Barbodes binotatus collected from Kerian River, Perak. The $a$ value ranged from 0.0011 for Barbodes binotatus, collected from Kerian River, Perak, to 0.0933 for Clarias batrachus collected from Pahang.

In Malaysia, Cyprinidae is the family most often studied for these LWRs, constituting of $46 \%$, followed by families of Clariidae and Pangasidae (6\%), Ambassidae, Bagridae and Sisoridae (5\%), Notoperidae and Clupeidae (3\%) and only $2 \%$ each for Cobitidae, Eleotrididae, Elopidae, Latidae, Megalopidae, Mugilidae, Pristolepididae, Scatophagidae, Schilbeidae, Siluridae and Tetraodontidae. Geographically, 94 (92\%) of the LWRs studies have been conducted in West Malaysia compared to East Malaysia, where only eight (8\%) studies have been reported. In West Malaysia, 50\% of LWR studies were conducted in the East Coast area (Pahang and Terengganu), while Northern states (Perak and Kedah) have contributed to 35\% of the studies. The Southern and West Coast area of West Malaysia remain the lowest at about $5 \%$ and $2 \%$, respectively. 
Table 1: Length-weight relationships of 64 freshwater fishes in Malaysia.

\begin{tabular}{|c|c|c|c|c|c|c|}
\hline Families & Species & Sampling area & $\mathrm{N}$ & $a$ & $b$ & References \\
\hline \multirow{3}{*}{ 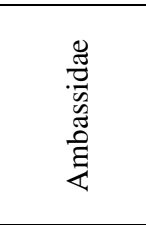 } & $\begin{array}{l}\text { Ambassis } \\
\text { interrupta }\end{array}$ & $\begin{array}{l}\text { Lutong River, } \\
\text { Sarawak }\end{array}$ & 118 & 0.0131 & 2.98 & Nyanti et al., 2012 \\
\hline & $\begin{array}{l}\text { Ambassis } \\
\text { kopsii }\end{array}$ & $\begin{array}{l}\text { Lutong River, } \\
\text { Sarawak }\end{array}$ & 43 & 0.0152 & 2.94 & Nyanti et al., 2012 \\
\hline & $\begin{array}{l}\text { Ambassis } \\
\text { urotaenia }\end{array}$ & $\begin{array}{l}\text { Lutong River, } \\
\text { Sarawak }\end{array}$ & 8 & 0.0214 & 2.65 & Nyanti et al., 2012 \\
\hline \multirow{3}{*}{ 营 } & $\begin{array}{l}\text { Arius } \\
\text { malculatus }\end{array}$ & $\begin{array}{l}\text { Pulai River, } \\
\text { Johor }\end{array}$ & 161 & 0.0295 & 2.62 & Arshad et al., 2008 \\
\hline & $\begin{array}{l}\text { Arius } \\
\text { tenuispinis }\end{array}$ & $\begin{array}{l}\text { Pulai River, } \\
\text { Johor }\end{array}$ & 70 & 0.0026 & 2.66 & Arshad et al., 2008 \\
\hline & $\begin{array}{l}\text { Plicofollis } \\
\text { argyropleuron }\end{array}$ & $\begin{array}{l}\text { Kuala Muda and Merbok, } \\
\text { Kedah }\end{array}$ & 539 & 0.0070 & 3.09 & $\begin{array}{l}\text { Nor Aziella and } \\
\text { Mansor, } 2012\end{array}$ \\
\hline \multirow{5}{*}{ 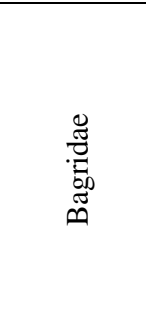 } & $\begin{array}{l}\text { Batasio } \\
\text { fluviatilis }\end{array}$ & $\begin{array}{l}\text { Pelus River, } \\
\text { Kuala Kangsar }\end{array}$ & 28 & 0.0323 & 2.44 & $\begin{array}{l}\text { Ikhwanuddin et al., } \\
2016\end{array}$ \\
\hline & \multirow[t]{3}{*}{$\begin{array}{l}\text { Hemibagrus } \\
\text { nemurus }\end{array}$} & $\begin{array}{l}\text { Pahang River, } \\
\text { Temerloh }\end{array}$ & 20 & 0.0068 & 3.07 & Zulkafli et al., 2015 \\
\hline & & Perak* & 30 & 0.0052 & 3.15 & Yusof et al., 2011 \\
\hline & & $\begin{array}{l}\text { Tembeling River, } \\
\text { Pahang }\end{array}$ & 47 & 0.0064 & 3.09 & Zulkafli et al., 2016 \\
\hline & $\begin{array}{l}\text { Pseudomystus } \\
\text { siamensis }\end{array}$ & Negeri Sembilan & 30 & 0.0060 & 3.20 & Yusof et al., 2011 \\
\hline \multirow{4}{*}{ 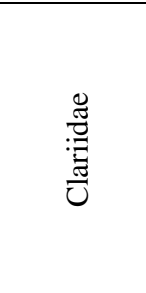 } & $\begin{array}{l}\text { Clarias } \\
\text { batrachus }\end{array}$ & Pahang* & 30 & 0.0933 & 2.19 & Yusof et al., 2011 \\
\hline & $\begin{array}{l}\text { Clarias } \\
\text { gariepinus }\end{array}$ & Selangor* & 30 & 0.0138 & 2.76 & Yusof et al., 2011 \\
\hline & $\begin{array}{l}\text { Clarias } \\
\text { leiacanthus }\end{array}$ & $\begin{array}{l}\text { Pahang River, } \\
\text { Maran }\end{array}$ & 34 & 0.0192 & 2.75 & Zulkafli et al., 2014 \\
\hline & $\begin{array}{l}\text { Clarias } \\
\text { macrocephalus }\end{array}$ & Pahang* & 30 & 0.0151 & 2.79 & Yusof et al., 2011 \\
\hline \multirow{2}{*}{ 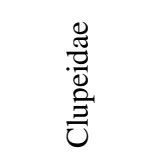 } & $\begin{array}{l}\text { Anodontostoma } \\
\text { chacunda }\end{array}$ & $\begin{array}{l}\text { Pulai River, } \\
\text { Johor }\end{array}$ & 133 & 0.0118 & 3.09 & Arshad et al., 2008 \\
\hline & Hilsa kelee & $\begin{array}{l}\text { Pulai River, } \\
\text { Johor }\end{array}$ & 499 & 0.0448 & 2.54 & Arshad et al., 2008 \\
\hline 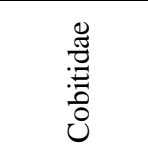 & $\begin{array}{l}\text { Acantopsis } \\
\text { dialuzona }\end{array}$ & $\begin{array}{l}\text { Kerian River basin, } \\
\text { Perak }\end{array}$ & 56 & 0.0190 & 2.43 & $\begin{array}{l}\text { Mohd-Shafiq et al., } \\
2012\end{array}$ \\
\hline \multirow{5}{*}{ 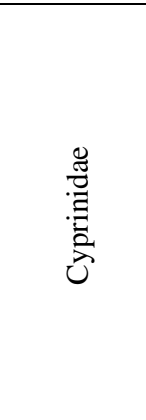 } & \multirow[t]{2}{*}{$\begin{array}{l}\text { Amblyrhynchicthys } \\
\text { truncatus }\end{array}$} & $\begin{array}{l}\text { Pahang River, } \\
\text { Maran }\end{array}$ & 175 & 0.0047 & 3.31 & Zulkafli et al., 2014 \\
\hline & & $\begin{array}{l}\text { Pahang River, } \\
\text { Temerloh }\end{array}$ & 14 & 0.0084 & 3.08 & Zulkafli et al., 2015 \\
\hline & $\begin{array}{l}\text { Anematichthys } \\
\text { repasson }\end{array}$ & $\begin{array}{l}\text { Tembeling River, } \\
\text { Pahang }\end{array}$ & 10 & 0.0063 & 3.19 & Zulkafli et al., 2016 \\
\hline & \multirow[t]{2}{*}{$\begin{array}{l}\text { Barbichthys } \\
\text { laevis }\end{array}$} & $\begin{array}{l}\text { Pahang River, } \\
\text { Maran }\end{array}$ & 98 & 0.0124 & 2.90 & Zulkafli et al., 2014 \\
\hline & & $\begin{array}{l}\text { Tembeling River, } \\
\text { Pahang }\end{array}$ & 60 & 0.0059 & 3.13 & Zulkafli et al., 2016 \\
\hline
\end{tabular}


Table 1 (continued): Length-weight relationships of 64 freshwater fishes in Malaysia.

\begin{tabular}{|c|c|c|c|c|c|c|}
\hline Families & Species & Sampling area & $\mathrm{N}$ & $\mathrm{a}$ & $\mathrm{b}$ & References \\
\hline \multirow{25}{*}{ 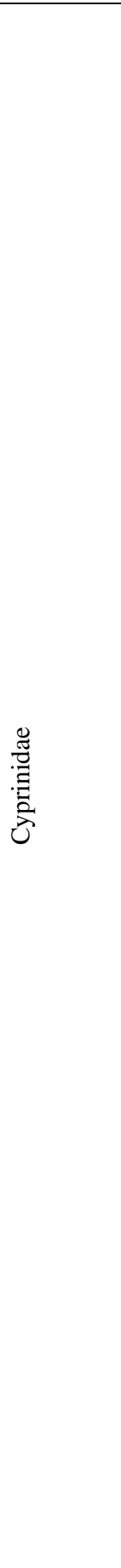 } & \multirow[t]{4}{*}{$\begin{array}{l}\text { Barbodes } \\
\text { binotatus }\end{array}$} & $\begin{array}{l}\text { Kerian River, } \\
\text { Perak }\end{array}$ & 76 & 0.0011 & 4.11 & Isa et al., 2010 \\
\hline & & $\begin{array}{l}\text { Langkawi Island, } \\
\text { Perak }\end{array}$ & 49 & 0.0130 & 2.99 & Samat et al., 2012 \\
\hline & & $\begin{array}{l}\text { Kerian River basin, } \\
\text { Perak }\end{array}$ & 92 & 0.0500 & 3.81 & $\begin{array}{l}\text { Mohd-Shafiq et al., } \\
2012\end{array}$ \\
\hline & & $\begin{array}{l}\text { Pelus River, } \\
\text { Kuala Kangsar }\end{array}$ & 16 & 0.0062 & 3.30 & $\begin{array}{l}\text { Ikhwanuddin et al., } \\
2016\end{array}$ \\
\hline & $\begin{array}{l}\text { Barbonymus } \\
\text { gonionotus }\end{array}$ & $\begin{array}{l}\text { Pedu Lake, } \\
\text { Kedah }\end{array}$ & 32 & 0.0058 & 3.23 & Isa et al., 2010 \\
\hline & \multirow[t]{4}{*}{$\begin{array}{l}\text { Barbonymus } \\
\text { schwanenfeldii }\end{array}$} & $\begin{array}{l}\text { Pedu Lake, } \\
\text { Kedah }\end{array}$ & 246 & 0.0168 & 2.86 & Isa et al., 2010 \\
\hline & & $\begin{array}{l}\text { Pahang River, } \\
\text { Maran }\end{array}$ & 145 & 0.0112 & 3.08 & Zulkafli et al., 2014 \\
\hline & & $\begin{array}{l}\text { Tembeling River, } \\
\text { Pahang }\end{array}$ & 124 & 0.0162 & 2.93 & Zulkafli et al., 2016 \\
\hline & & $\begin{array}{l}\text { Pahang River, } \\
\text { Temerloh }\end{array}$ & 88 & 0.0141 & 3.00 & Zulkafli et al., 2015 \\
\hline & Chela sp. & $\begin{array}{l}\text { Pedu Lake, } \\
\text { Kedah }\end{array}$ & 88 & 0.0158 & 2.67 & Isa et al., 2010 \\
\hline & $\begin{array}{l}\text { Cirrhinus } \\
\text { caudimaculatus }\end{array}$ & $\begin{array}{l}\text { Tembeling River, } \\
\text { Pahang }\end{array}$ & 152 & 0.0067 & 3.08 & Zulkafli et al., 2016 \\
\hline & $\begin{array}{l}\text { Crossocheilus } \\
\text { atrilimes }\end{array}$ & $\begin{array}{l}\text { Pelus River, } \\
\text { Kuala Kangsar }\end{array}$ & 36 & 0.0062 & 3.09 & $\begin{array}{l}\text { Ikhwanuddin et al., } \\
2016\end{array}$ \\
\hline & \multirow[t]{3}{*}{$\begin{array}{l}\text { Cyclocheilichthys } \\
\text { apogon }\end{array}$} & $\begin{array}{l}\text { Kerian River, } \\
\text { Perak }\end{array}$ & 46 & 0.0033 & 3.51 & Isa et al., 2010 \\
\hline & & $\begin{array}{l}\text { Kerian River basin, } \\
\text { Perak }\end{array}$ & 56 & 0.0020 & 3.63 & $\begin{array}{l}\text { Mohd-Shafiq et al., } \\
2012\end{array}$ \\
\hline & & $\begin{array}{l}\text { Temengor Reservoir, } \\
\text { Perak }\end{array}$ & 233 & 0.0080 & 3.16 & $\begin{array}{l}\text { Muzzalifah et al., } \\
2015\end{array}$ \\
\hline & \multirow[t]{3}{*}{$\begin{array}{l}\text { Cyclocheilichthys } \\
\text { apogon }\end{array}$} & $\begin{array}{l}\text { Pahang River, } \\
\text { Maran }\end{array}$ & 203 & 0.0071 & 3.22 & Zulkafli et al., 2014 \\
\hline & & $\begin{array}{l}\text { Tembeling River, } \\
\text { Pahang }\end{array}$ & 215 & 0.0072 & 3.15 & Zulkafli et al., 2016 \\
\hline & & $\begin{array}{l}\text { Pahang River, } \\
\text { Temerloh }\end{array}$ & 48 & 0.0095 & 3.05 & Zulkafli et al., 2015 \\
\hline & \multirow[t]{4}{*}{$\begin{array}{l}\text { Devario } \\
\text { regina }\end{array}$} & $\begin{array}{l}\text { Pelus River, } \\
\text { Kuala Kangsar }\end{array}$ & 58 & 0.0132 & 2.82 & $\begin{array}{l}\text { Ikhwanuddin et al., } \\
2016\end{array}$ \\
\hline & & $\begin{array}{l}\text { Kerian River, } \\
\text { Perak }\end{array}$ & 112 & 0.0057 & 3.3 & Isa et al., 2010 \\
\hline & & $\begin{array}{l}\text { Langkawi Island, } \\
\text { Perak }\end{array}$ & 52 & 0.0080 & 3.06 & Samat et al., 2012 \\
\hline & & $\begin{array}{l}\text { Kerian River basin, } \\
\text { Perak }\end{array}$ & 124 & 0.0130 & 3.25 & $\begin{array}{l}\text { Mohd-Shafiq et al., } \\
2012\end{array}$ \\
\hline & \multirow[t]{3}{*}{$\begin{array}{l}\text { Hampala } \\
\text { macrolepidota }\end{array}$} & $\begin{array}{l}\text { Kenyir Lake, } \\
\text { Terengganu }\end{array}$ & 2057 & 0.0148 & 2.88 & Zakaria et al., 2000 \\
\hline & & $\begin{array}{l}\text { Temengor Reservoir, } \\
\text { Perak }\end{array}$ & 207 & 0.0100 & 3.02 & $\begin{array}{l}\text { Muzzalifah et al., } \\
2015\end{array}$ \\
\hline & & $\begin{array}{l}\text { Tembeling River, } \\
\text { Pahang }\end{array}$ & 23 & 0.0105 & 2.99 & Zulkafli et al., 2016 \\
\hline
\end{tabular}


Table 1 (continued): Length-weight relationships of 64 freshwater fishes in Malaysia.

\begin{tabular}{|c|c|c|c|c|c|c|}
\hline Families & Species & Sampling area & $\mathrm{N}$ & $a$ & $b$ & References \\
\hline \multirow{26}{*}{ 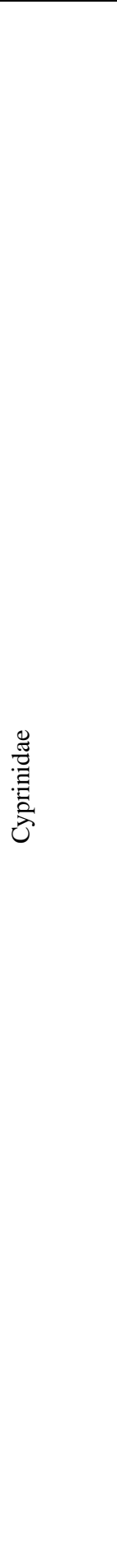 } & $\begin{array}{l}\text { Hypsibarbus } \\
\text { pierrei }\end{array}$ & $\begin{array}{l}\text { Pahang River, } \\
\text { Maran }\end{array}$ & 60 & 0.0115 & 3.03 & Zulkafli et al., 2014 \\
\hline & \multirow[t]{3}{*}{$\begin{array}{l}\text { Hypsibarbus } \\
\text { wetmorei }\end{array}$} & $\begin{array}{l}\text { Pahang River, } \\
\text { Maran }\end{array}$ & 231 & 0.0079 & 3.15 & Zulkafli et al., 2014 \\
\hline & & $\begin{array}{l}\text { Tembeling River, } \\
\text { Pahang }\end{array}$ & 19 & 0.0118 & 3.01 & Zulkafli et al., 2016 \\
\hline & & $\begin{array}{l}\text { Pahang River, } \\
\text { Temerloh }\end{array}$ & 49 & 0.0094 & 3.11 & Zulkafli et al., 2015 \\
\hline & $\begin{array}{l}\text { Labiobarbus } \\
\text { festivus }\end{array}$ & $\begin{array}{l}\text { Pahang River, } \\
\text { Temerloh }\end{array}$ & 56 & 0.00892 & 3.00 & Zulkafli et al., 2015 \\
\hline & & $\begin{array}{l}\text { Tembeling River, } \\
\text { Pahang }\end{array}$ & 103 & 0.0072 & 3.07 & Zulkafli et al., 2016 \\
\hline & \multirow[t]{2}{*}{$\begin{array}{l}\text { Labiobarbus } \\
\text { leptocheilus }\end{array}$} & $\begin{array}{l}\text { Kerian River basin, } \\
\text { Perak }\end{array}$ & 104 & 0.0280 & 2.86 & $\begin{array}{l}\text { Mohd-Shafiq et al., } \\
2012\end{array}$ \\
\hline & & $\begin{array}{l}\text { Temengor Reservoir, } \\
\text { Perak }\end{array}$ & 73 & 0.0150 & 2.85 & $\begin{array}{l}\text { Muzzalifah et al., } \\
2015\end{array}$ \\
\hline & $\begin{array}{l}\text { Labiobarbus } \\
\text { lineatus }\end{array}$ & Kerian River, Perak & 58 & 0.0095 & 2.99 & Isa et al., 2010 \\
\hline & $\begin{array}{l}\text { Luciosoma } \\
\text { setigerum }\end{array}$ & $\begin{array}{l}\text { Pahang River, } \\
\text { Temerloh }\end{array}$ & 9 & 0.0088 & 2.86 & Zulkafli et al., 2015 \\
\hline & $\begin{array}{l}\text { Mystacoleucus } \\
\text { marginatus }\end{array}$ & $\begin{array}{l}\text { Tembeling River, } \\
\text { Pahang }\end{array}$ & 32 & 0.0074 & 3.17 & Zulkafli et al., 2016 \\
\hline & \multirow[t]{3}{*}{$\begin{array}{l}\text { Mystacoleucus } \\
\text { obtusirostris }\end{array}$} & $\begin{array}{l}\text { Pedu Lake, } \\
\text { Kedah }\end{array}$ & 65 & 0.0230 & 2.70 & Isa et al., 2010 \\
\hline & & $\begin{array}{l}\text { Pahang River, } \\
\text { Maran }\end{array}$ & 31 & 0.0137 & 2.94 & Zulkafli et al., 2014 \\
\hline & & $\begin{array}{l}\text { Temengor Reservoir, } \\
\text { Perak }\end{array}$ & 83 & 0.0070 & 3.15 & $\begin{array}{l}\text { Muzzalifah et al., } \\
2015\end{array}$ \\
\hline & $\begin{array}{l}\text { Neolissochilus } \\
\text { hexagonolepis }\end{array}$ & $\begin{array}{l}\text { Pelus River, } \\
\text { Kuala Kangsar }\end{array}$ & 128 & 0.0099 & 3.04 & $\begin{array}{l}\text { Ikhwanuddin et al., } \\
2016\end{array}$ \\
\hline & $\begin{array}{l}\text { Osteochilus } \\
\text { microcephalus }\end{array}$ & $\begin{array}{l}\text { Pedu Lake, } \\
\text { Kedah }\end{array}$ & 35 & 0.0075 & 3.05 & Isa et al., 2010 \\
\hline & \multirow[t]{4}{*}{$\begin{array}{l}\text { Osteochilus } \\
\text { vittatus }\end{array}$} & $\begin{array}{l}\text { Temengor Reservoir, } \\
\text { Perak }\end{array}$ & 357 & 0.0100 & 3.04 & $\begin{array}{l}\text { Muzzalifah et al., } \\
2015\end{array}$ \\
\hline & & $\begin{array}{l}\text { Pahang River, } \\
\text { Maran }\end{array}$ & 89 & 0.0093 & 3.12 & Zulkafli et al., 2014 \\
\hline & & $\begin{array}{l}\text { Tembeling River, } \\
\text { Pahang }\end{array}$ & 10 & 0.0168 & 2.85 & Zulkafli et al., 2016 \\
\hline & & $\begin{array}{l}\text { Pahang River, } \\
\text { Temerloh }\end{array}$ & 19 & 0.0137 & 2.93 & Zulkafli et al., 2015 \\
\hline & $\begin{array}{l}\text { Osteochilus } \\
\text { waandersii }\end{array}$ & $\begin{array}{l}\text { Pahang River, } \\
\text { Temerloh }\end{array}$ & 24 & 0.0075 & 3.12 & Zulkafli et al., 2015 \\
\hline & $\begin{array}{l}\text { Oxygaster } \\
\text { anomalura }\end{array}$ & $\begin{array}{l}\text { Temengor Reservoir, } \\
\text { Perak }\end{array}$ & 171 & 0.0200 & 2.60 & $\begin{array}{l}\text { Muzzalifah et al., } \\
2015\end{array}$ \\
\hline & \multirow[t]{2}{*}{$\begin{array}{l}\text { Puntioplites } \\
\text { bulu }\end{array}$} & $\begin{array}{l}\text { Pahang River, } \\
\text { Maran }\end{array}$ & 43 & 0.0108 & 3.04 & Zulkafli et al., 2014 \\
\hline & & $\begin{array}{l}\text { Tembeling River, } \\
\text { Pahang }\end{array}$ & 18 & 0.0119 & 3.01 & Zulkafli et al., 2016 \\
\hline & $\begin{array}{l}\text { Puntioplites } \\
\text { proctozysron }\end{array}$ & $\begin{array}{l}\text { Tembeling River, } \\
\text { Pahang }\end{array}$ & 241 & 0.0087 & 3.15 & Zulkafli et al., 2016 \\
\hline & $\begin{array}{l}\text { Probarbus } \\
\text { jullieni }\end{array}$ & $\begin{array}{l}\text { Pahang River, } \\
\text { Maran }\end{array}$ & 89 & 0.0080 & 3.09 & Zulkafli et al., 2014 \\
\hline
\end{tabular}


Table 1 (continued): Length-weight relationships of 64 freshwater fishes in Malaysia.

\begin{tabular}{|c|c|c|c|c|c|c|}
\hline Families & Species & Sampling area & $\mathrm{N}$ & $a$ & $b$ & References \\
\hline \multirow{4}{*}{ 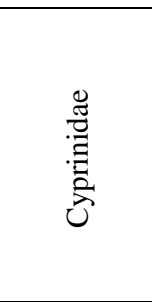 } & \multirow[t]{2}{*}{\begin{tabular}{|l} 
Rasbora \\
sumatrana
\end{tabular}} & $\begin{array}{l}\text { Kerian River, } \\
\text { Perak }\end{array}$ & 77 & 0.0025 & 3.61 & Isa et al., 2010 \\
\hline & & $\begin{array}{l}\text { Kerian River basin, } \\
\text { Perak }\end{array}$ & 92 & 0.0110 & 3.30 & $\begin{array}{l}\text { Mohd-Shafiq et al., } \\
2012\end{array}$ \\
\hline & \multirow[t]{2}{*}{$\begin{array}{l}\text { Thynnichthys } \\
\text { thynnoides }\end{array}$} & $\begin{array}{l}\text { Pahang River, } \\
\text { Temerloh }\end{array}$ & 43 & 0.0114 & 2.92 & Zulkafli et al., 2015 \\
\hline & & $\begin{array}{l}\text { Pahang River, } \\
\text { Maran }\end{array}$ & 52 & 0.0063 & 3.13 & Zulkafli et al., 2014 \\
\hline 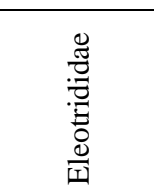 & $\begin{array}{l}\text { Oxyeleotris } \\
\text { marmorata }\end{array}$ & $\begin{array}{l}\text { Pahang River, } \\
\text { Maran }\end{array}$ & 30 & 0.0049 & 3.28 & Zulkafli et al., 2014 \\
\hline 鰙 & $\begin{array}{l}\text { Lates } \\
\text { calcarifer }\end{array}$ & $\begin{array}{l}\text { Lutong River, } \\
\text { Sarawak }\end{array}$ & 10 & 0.0144 & 3.02 & Nyanti et al., 2012 \\
\hline $\begin{array}{l}\frac{\pi}{0} \\
: 0 \\
: 0 \\
\stackrel{0}{0}\end{array}$ & $\begin{array}{l}\text { Liza } \\
\text { melinoptera }\end{array}$ & $\begin{array}{l}\text { Lutong River, } \\
\text { Sarawak }\end{array}$ & 8 & 0.0149 & 2.95 & Nyanti et al., 2012 \\
\hline 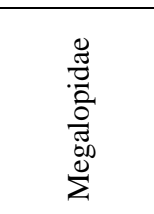 & $\begin{array}{l}\text { Megalops } \\
\text { cyprinoides }\end{array}$ & $\begin{array}{l}\text { Lutong River, } \\
\text { Sarawak }\end{array}$ & 24 & 0.0173 & 2.79 & Nyanti et al., 2012 \\
\hline \multirow{2}{*}{ 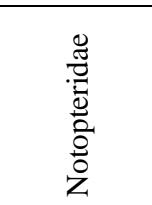 } & $\begin{array}{l}\text { Chitala } \\
\text { Lopis }\end{array}$ & $\begin{array}{l}\text { Pahang River, } \\
\text { Maran }\end{array}$ & 33 & 0.0173 & 2.69 & Zulkafli et al., 2014 \\
\hline & $\begin{array}{l}\text { Notopterus } \\
\text { Notopterus }\end{array}$ & $\begin{array}{l}\text { Pedu Lake, } \\
\text { Kedah }\end{array}$ & 120 & 0.0036 & 3.25 & Isa et al., 2010 \\
\hline \multirow{7}{*}{ 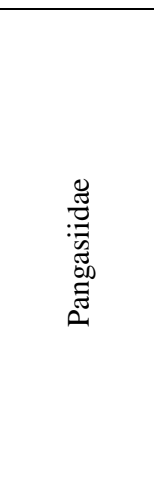 } & $\begin{array}{l}\text { Pangasianodon } \\
\text { hypophthalmus }\end{array}$ & $\begin{array}{l}\text { Pahang River, } \\
\text { Maran }\end{array}$ & 24 & 0.0227 & 2.75 & Zulkafli et al., 2014 \\
\hline & $\begin{array}{l}\text { Pangasius } \\
\text { pangasius }\end{array}$ & Perak* & 30 & 0.0290 & 2.72 & Yusof et al., 2011 \\
\hline & \multirow{2}{*}{$\begin{array}{l}\text { Pangasius } \\
\text { nasutus }\end{array}$} & Selangor* & 30 & 0.0479 & 2.57 & Yusof et al., 2011 \\
\hline & & $\begin{array}{l}\text { Pahang River, } \\
\text { Maran }\end{array}$ & 24 & 0.0045 & 3.16 & Zulkafli et al., 2014 \\
\hline & \multirow[t]{3}{*}{$\begin{array}{l}\text { Pseudolais } \\
\text { micronemus }\end{array}$} & $\begin{array}{l}\text { Pahang River, } \\
\text { Maran }\end{array}$ & 46 & 0.0069 & 3.02 & Zulkafli et al., 2014 \\
\hline & & $\begin{array}{l}\text { Pahang River, } \\
\text { Temerloh }\end{array}$ & 33 & 0.0071 & 3.00 & Zulkafli et al., 2015 \\
\hline & & $\begin{array}{l}\text { Tembeling River, } \\
\text { Pahang }\end{array}$ & 143 & 0.0105 & 2.88 & Zulkafli et al., 2016 \\
\hline \multirow{2}{*}{ 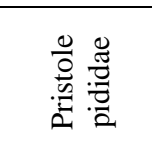 } & \multirow[t]{2}{*}{$\begin{array}{l}\text { Pristolepis } \\
\text { fasciata }\end{array}$} & $\begin{array}{l}\text { Temengor Reservoir, } \\
\text { Perak }\end{array}$ & 31 & 0.0180 & 3.07 & Yusof et al., 2011 \\
\hline & & $\begin{array}{l}\text { Pahang River, } \\
\text { Maran }\end{array}$ & 43 & 0.0160 & 3.12 & Zulkafli et al., 2014 \\
\hline
\end{tabular}


Table 1 (continued): Length-weight relationships of 64 freshwater fishes in Malaysia; note: * denotes no specific area reported by authors.

\begin{tabular}{|c|c|c|c|c|c|c|}
\hline Families & Species & Sampling area & $\mathrm{N}$ & $a$ & $b$ & References \\
\hline \multirow[t]{2}{*}{ Pristolepididae } & \multirow[t]{2}{*}{$\begin{array}{l}\text { Pristolepis } \\
\text { fasciata }\end{array}$} & $\begin{array}{l}\text { Temengor } \\
\text { Reservoir, } \\
\text { Perak }\end{array}$ & 31 & 0.0180 & 3.07 & Yusof et al., 2011 \\
\hline & & $\begin{array}{l}\text { Pahang River, } \\
\text { Maran }\end{array}$ & 43 & 0.0160 & 3.12 & Zulkafli et al., 2014 \\
\hline Scatophagidae & $\begin{array}{l}\text { Scatophagus } \\
\text { argus }\end{array}$ & $\begin{array}{l}\text { Lutong River, } \\
\text { Sarawak }\end{array}$ & 16 & 0.0359 & 3.03 & Nyanti et al., 2012 \\
\hline \multirow{2}{*}{ Schilbeidae } & \multirow[t]{2}{*}{$\begin{array}{l}\text { Laides } \\
\text { hexanema }\end{array}$} & $\begin{array}{l}\text { Pahang River, } \\
\text { Temerloh }\end{array}$ & 17 & 0.0070 & 3.09 & Zulkafli et al., 2015 \\
\hline & & $\begin{array}{l}\text { Pahang River, } \\
\text { Maran }\end{array}$ & 120 & 0.0043 & 3.24 & Zulkafli et al., 2014 \\
\hline \multirow{2}{*}{ Siluridae } & \multirow[t]{2}{*}{$\begin{array}{l}\text { Phalacronotus } \\
\text { apogon }\end{array}$} & $\begin{array}{l}\text { Pahang River, } \\
\text { Maran }\end{array}$ & 49 & 0.0258 & 2.43 & Zulkafli et al., 2014 \\
\hline & & $\begin{array}{l}\text { Tembeling River, } \\
\text { Pahang }\end{array}$ & 25 & 0.0049 & 3.08 & Zulkafli et al., 2016 \\
\hline \multirow{3}{*}{ Sisoridae } & $\begin{array}{l}\text { Bagarius } \\
\text { yarrelli }\end{array}$ & $\begin{array}{l}\text { Pahang River, } \\
\text { Temerloh }\end{array}$ & 14 & 0.0146 & 2.72 & Zulkafli et al., 2015 \\
\hline & $\begin{array}{l}\text { Glyptothorax } \\
\text { major }\end{array}$ & $\begin{array}{l}\text { Pelus River, } \\
\text { Kuala Kangsar }\end{array}$ & 62 & 0.0074 & 3.15 & $\begin{array}{l}\text { Ikhwanuddin et al., } \\
2016\end{array}$ \\
\hline & $\begin{array}{l}\text { Glyptothorax } \\
\text { siamensis }\end{array}$ & $\begin{array}{l}\text { Pelus River, } \\
\text { Kuala Kangsar }\end{array}$ & 23 & 0.0089 & 2.98 & $\begin{array}{l}\text { Ikhwanuddin et al., } \\
2016\end{array}$ \\
\hline Tetraodontidae & $\begin{array}{l}\text { Arothron } \\
\text { reticularis }\end{array}$ & $\begin{array}{l}\text { Lutong River, } \\
\text { Sarawak }\end{array}$ & 9 & 0.0359 & 2.95 & Nyanti et al., 2012 \\
\hline
\end{tabular}

From a total of 13 conducted previous studies, it can be listed that 32 fish species from 47 LWR studies experienced positive allometric growth, where 20 fish species from 23 LWR studies experienced isometric growth and 28 fish species from 31 LWR studies experienced negative allometric growth (Fig. 1). However, there were also different types of growth suggested by researchers for the same species. For instance, the growth pattern of Barbodes binotatus was isometric in Langkawi Island as reported by Samat et al. (2012), but Isa et al. (2010) and Mohd-Shafiq et al. (2010) recorded positive allometric growth for this species. These observations were also identified for 12 more species, such as Barbonymus schwanenfeldii, Barbichthys laevis, Labiobarbus festivus, Thynnichthys thynnoides, Devario regina, Hypsibarbus wetmorei, Pseudolais micronemus, Hampala macrolepidota, Mystacoleucus obtusirostris, Osteochilus vittatus, Phalacronotus apogon and Pangasius nasutus. These findings might be due to different habitat conditions in terms of variations of food supply which influences the habitual preferences of the fish, fish activities, and feeding habits of fish species ( Mizuno and Furtado, 1982; Lowe-McConnell, 1987). 


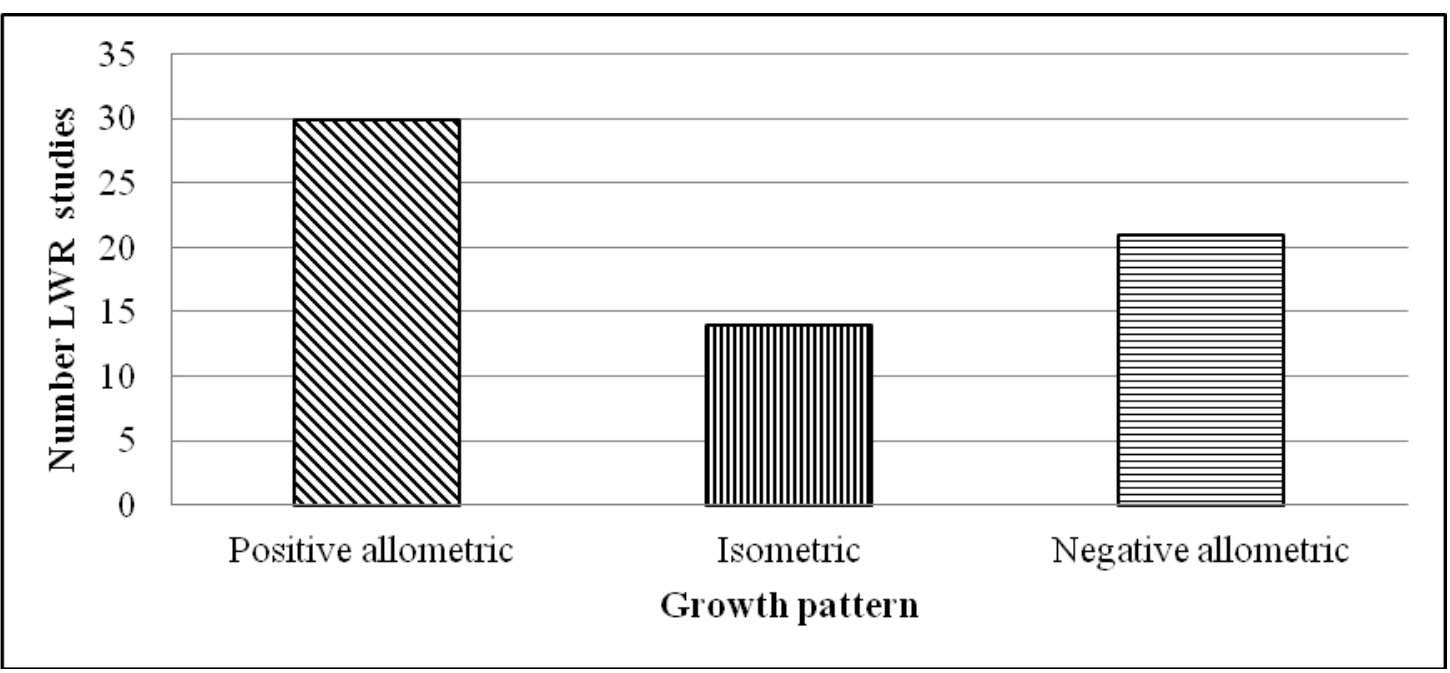

Figure 1: Growth patterns of 64 freshwater fishes

from 102 length-weight relationships studies in Malaysia.

The mean for $a$ value from 64 LWRs was 0.0136, and ranging from 0.0011 for Barbodes binotatus collected in Kerian River, Perak, to 0.0933 for Clarias batrachus collected in Pahang. By referring to Froese and Pauly (2015), body type of all 64 species were determined (Tab. 2). From 64 species, there were 11 (17\%) species that have elongated body type, 30 (47\%) have fusiform body type and the other 22 (36\%) species showed short and deep body type (Fig. 2). In term of the fish families, there were 15 families that exhibit short and deep body type, eight have fusiform and only six have elongated body type. There were three families (Aaridae, Cyprinidae and Sisoridae) that have multiple body types for different species of fishes. Eel-like body type is absent from the result because there was no $a$ value that fit in the category (Tab. 2). It is important to note that fusiform is the most general body type and ideal for free swimming species (Khanna, 2004), compared to other body types. Helfman et al. (2009) stated that body type is one of the factors that help fish swim faster, thus determining their feeding habits and desired habitat.

The mean of $b$ value for 64 LWR studies of 49 freshwater fishes in Malaysia was 3.0139, which indicated that their growth is still in the normal range for freshwater fish. Froese (2006) has confirmed that expected range of $b$ is $2.5<b<3.5$. From the results, it is also necessary to study more about negative allometric growth (20 species), whether they are genuinely affected by environmental condition or statistical inaccuracy due to inadequate LWR data collection. However, according to Copp et al. (2013), the isometric body growth of Barbatula barbatula changes to allometric type when it reaches a certain standard length in order to become an adult. This indicates that body growth type sometimes changes accordingly to their physiology needs. 
Low catch of fish species may be contributed by the unsuitable fishing gear used or inadequate and unfit timing. The number of sample size also affects the quality of the relationships. Only $n>10$ should be accepted because Central Limit Theorem has been used. Also note that sampling distribution of the sample mean will approximately become normal as the sample size increases. Thus, the value of $n$ below 10 will not provide a good approximation to the probability of interest (Kenneth, 2007). It is important to note that there are 14 LWRs that have regression value below than $0.9\left(R^{2}<0.9\right)$. For example, Ambassis urotaenia have very small sample size $(n=8)$, which could have answered its low regression value $\left(R^{2}=\right.$ 0.850) and considered statistically low for LWR study. In contrast, even a total of 70 individuals of Arius tenuispinis have been recorded, but the $R^{2}$ value is only recorded at 0.850 . The condition is same with Chela sp., Devario regina, Mystacoleucus obstusirostris, Osteochilus microchepalus, Lates calcarifer, Megalops cyprinoides, Rasbora sumatrana, Pangasius pangasius and Pangasius nasutus. Moreover, even though sample size for Luciosoma setigerum, Liza melinoptera and Arothron reticularis are low $(n<10)$, the $R^{2}$ value is significantly strong. Kelley and Preacher (2012) stated that these observation sometimes due to a small sized sample is sometimes considered enough for certain fish species. Besides that, possibilities of human error (data collection, recording or entry) and inconsideration of outliers in the LWR calculation (Osborne et al., 2004) also makes the $R^{2}$ value lower, resulting in less confidence in the information.

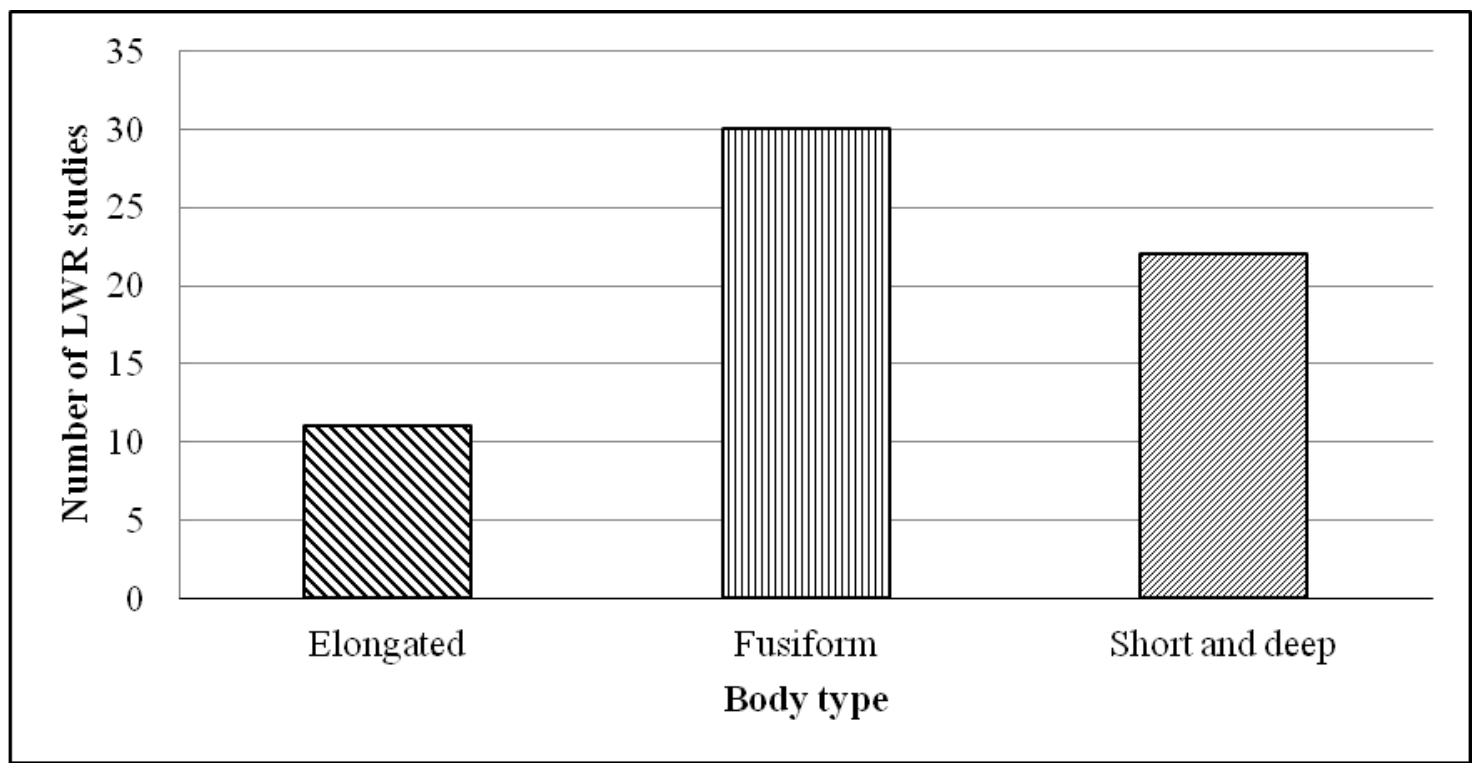

Figure 2: Body type of 64 freshwater fishes from 102 length-weight relationships studies in Malaysia. 
Table 2: Estimation of body shape mean by following Bayesian length-weight relationship (Froese and Pauly, 2015).

\begin{tabular}{|l|c|c|}
\hline $\begin{array}{c}\text { Body } \\
\text { shape }\end{array}$ & $\begin{array}{c}\text { Prior for } b \\
\text { (mean } \pm \text { SD) }\end{array}$ & $\begin{array}{c}\text { Prior of log10(a) } \\
\text { (mean } \pm \text { SD) }\end{array}$ \\
\hline Eel-like & $3.06 \pm 0.0896$ & $-2.99 \pm 0.175$ \\
\hline Elongated & $3.12 \pm 0.09$ & $-2.41 \pm 0.171$ \\
\hline Fusiform & $3.04 \pm 0.0857$ & $-1.95 \pm 0.173$ \\
\hline Short and deep & $3.01 \pm 0.0905$ & $-1.7 \pm 0.175$ \\
\hline
\end{tabular}

Ten fish species that have three or more LWRs were chosen for comparison with FishBase Bayesian database in order to analyse the similarity of $a$ and $b$ value average and range between both methods. Only four species, namely Hemibagrus nemurus, Hampala macropleidata, Mystacoleucus obstusirostris, and Osteochilus vittatus, were available in the FishBase database. From the four species, only Hemibagrus nemurus observed within the Bayesian LWR range (Tab. 3). This condition may be related to lack of data of those species to calculate the range more accurately. Bayesian LWR needs a significant number of baseline data to create a meaningful mean of 95\% High Density Interval of $a$ and $b$ values (Froese et al., 2014). Unfortunately, Cole-Fletcher et al. (2011) have observed some flaw on FishBase's LWR database where studied species minimum and maximum curves produced with the length-weight parameters at FishBase.org are notably different from each other, and in many cases predict weights that are clearly absurd. Hence, to utilize FishBase database, a few statistical tests and corrections need to be conducted to ensure the accuracy of data presentation.

Table 3: Comparison of a and b values range between normal and Bayesian lengthweight relationship of four selected species.

\begin{tabular}{|l|l|l|l|l|c|}
\hline \multicolumn{1}{|c|}{ Species } & $\begin{array}{c}a \text { value } \\
\text { LWR range }\end{array}$ & $\begin{array}{c}\text { 95\% of HDI } \\
\text { of } a \text { value }\end{array}$ & $\begin{array}{c}b \text { value } \\
\text { LWR range }\end{array}$ & $\begin{array}{l}\text { 95\% of HDI } \\
\text { of } b \text { value }\end{array}$ & In range? \\
\hline Hemibagrus & $0.0052-$ & $0.00379-$ & $3.07-$ & $2.99-$ & Yes \\
nemurus & 0.00677 & 0.00978 & 3.151 & 3.26 & No \\
\hline Hampala & $0.0100-$ & $0.00907-$ & $2.884-$ & $2.92-$ & No \\
macropleidata & 0.148 & 0.0235 & 3.019 & 3.18 & No \\
\hline $\begin{array}{l}\text { Mystacoleucus } \\
\text { obstusirostris }\end{array}$ & $0.0070-$ & $0.00608-$ & $2.704-$ & $2.85-$ & 3.15 \\
\hline $\begin{array}{l}\text { Osteochilus } \\
\text { vittatus }\end{array}$ & 0.00930 & 0.0214 & 3.147 & $2.97-$ & No \\
\hline
\end{tabular}




\section{CONCLUSIONS}

The majority of freshwater fishes in Malaysia have fusiform body type based on mean value of $a$. The mean of $b$ value for 64 LWRs studies for 49 freshwater fishes in Malaysia was 3.0139. It showed that their growth is still in the normal range for freshwater fish. Thus, it can be concluded that growth pattern for freshwater fish in Malaysia can be considered good even though some changes in $b$ value within these fish species were identified. The fish LWRs observed can be used as an indicator of environmental changes and fish ecological health for freshwater fishes in Malaysia.

\section{ACKNOWLEDGEMENTS}

We would like to send our appreciation to members of Aquatic Laboratory, faculty of Environmental Studies, UPM for helping the team. This study was sponsored by UPM Putra Grant GPM/2013/9422500 (Universiti Putra Malaysia). 


\section{REFERENCES}

1. Adaka G., Ndukwe E. and Nlewadim A., 2015 - Length-weight relationship of some fish species in a tropical rainforest river in southeast Nigeria, Transylvanian Review of Systematical and Ecological Research, The Wetlands Diversity, 17.2, 73-78.

Arshad A., Jimmy A., Nurul Amin S. M., Japar Sidik B. and Harah Z. M., 2008 - Length-weight and length-length relationships of five fish species collected from seagrass beds of the Sungai Pulai estuary, Peninsular Malaysia, Journal of Applied Ichthyology, 24, 328-329.

2. Atama C. I., Okeke O. C., Ekeh F. N., Ezenwaji N. E., Onah I. E., Ivoke N. and Eyo J. E., 2013 - Length-weight relationship and condition factor of six cichlid (cichilidae, perciformes) species of Anambra River, Nigeria, Journal of Fisheries and Aquaculture, 4, 82-86.

3. Bagenal T. B. and Tesch F. W., 1978 - Aspects of fish fecundity in ecology of freshwater fish production, in Methods for assessment of fish production in freshwater, 3rd edition, Oxford, Blackwell Scientific Publications, 75-101.

4. Bowen Z. H., Bovee K. D. and Waddle T. J., 2003 - Effect of flow regulation on shallow water habit dynamics and floodplain connectivity, Transactions of the American Fisheries Society, 132, 809-823.

5. Chew P. C. and Zulkafli A. R., 2007 - Sperm cryopreservation of some freshwater fish species in Malaysia, in Current Frontiers in Cryopreservation, InTech, 269-292.

6. Chong V. C., Lee P. K. Y. and Lau C. M., 2010 - Diversity, extinction risk and conservation of Malaysian fishes, Journal of Fish Biology, 76, 2009-2066.

7. Cole-Fletcher S., Marin-Salcedo L., Rana A. and Courtney M., 2011 - Errors in Length-weight Parameters at FishBase.org, Nature Precedings, 1-7.

8. Copp G. H., Vladimir K. and Hensel K., 2013 - When do fishes become juveniles?, Developments in Environmental Biology of Fishes, 19, Springer Science and Business Media, 286.

9. Francis R. A., 2012 - A handbook of global freshwater invasive species, Oxon, Routledge, 456.

10. Froese R., 2006 - Cube law, condition factor and weight-length relationships: history, metaanalysis and recommendations, Journal of Applied Ichthyology, 22, 241-253.

11. Froese R. and Pauly D., 2015 - FishBase, Retrieved September 21, 2015, from www.fishbase.org

12. Froese R. and Pauly D., 2016 - FishBase, Retrieved January 21, 2016, from www.fishbase.org

13. Froese R., Thorson J. T. and Reyes R. B., 2014 - A Bayesian approach for estimating lengthweight relationships in fishes, Journal of Applied Ichthyology, 30, 78-85.

14. Helfman G. S., Collette B. B. and Facey D. E., 2009 - The diversity of fishes, West Sussex, United Kingdom, John Wiley and Sons, 736.

15. Hilborn E. and Walters C. J., 2001 - Quantitative fisheries stock assessment: choice, dynamics and uncertainty, New York, Chapman and Hall, 570.

16. Ikhwanuddin M. E. M., Amal M. N. A., Shohaimi S., Azizul A., Johari S., Abdullah T. and Jamil N. R., 2016 - Length-weight relationships of seven fish species from upper Perlus River, Kuala Kangsar district, Perak, Malaysia, Journal of Applied Ichthyology, 32, 511-512.

17. Isa M. M., Rawi C. S., Rosla R., Anuar S. and Shah M., 2010 - Length-weight relationships of freshwater fish species in Kerian River basin and Pedu Lake, Research Journal of Fisheries and Hydrobiology, 5, 1-8.

18. Jones R. E., Petrell R. J. and Pauly D., 1999 - Using modified length-weight realtionship to assess the condition of fish, Aquaculture Engineering, 20, 261-276.

19. Kelley K. and Preacher K. J., 2012 - On effect size, Psychological Methods, 17, 137-152.

20. Kenneth G. G., 2007 - Power and sample size estimation techniques for fisheries management, North American Journal of Fisheries Management, 27, 397-404.

21. Khanna D. R., 2004 - Biology of Fishes, Delhi, Discovery Publishing House, 400.

22. Kuikka S., Vanhatalo J., Pulkkinen H., Mantyniemi S. and Corander J., 2014 - Experiences in Bayesian inference in Baltic salmon management, Statistical Science, 29, 42-49. 
23. Lawson E. O., 2011 - Length-weight relationships and fecundity estimates in mud-skipper, Periopthalmus papilio (Bloch and Schneider, 1801) caught form the mangrove swamps of Lagos Lagoon, Nigeria, Journal of Fisheries and Aquatic Science, 6, 264-271.

24. Lim K. K. P. and Tan H. H., 2002 - Freshwater fish diversity in Peninsular Malaysia: A review of recent findings, Penang, Penerbit Universiti Sains Malaysia, in Ali A., Rawi C. S. M., Mansor M., Nakamura R., Ramakrishnan S., and Mundkur T. (eds), Proceedings of the Asian Wetland Symposium, 2001, The Asian wetlands: Bringing partnerships into good wetland practices, Pulau Pinang, Penerbit, University Sains Malaysia, 461-481.

25. Lowe-McConnell R. H., 1987 - Ecological studies in tropical fish communities, Cambridge: Cambridge University Press, 400.

26. Mizuno N. and Furtado J. I., 1982 - Ecological notes on fishes in Tasik Bera, The Ecology of Freshwater Swamp, Netherlands, Junk W., 321-354.

27. Muzzalifah A. H., Mashhor M. and Siti Azizah M., 2015 - Length-weight relationship and condition factor of fish populations in Temengor Reservoir, Sains Malaysiana, 44, 61-66.

28. Nor Aziella M. R. and Mansor M. I., 2012 - Length-weight and length-length relationship of Longsnouted Catfish, Plicofollis argyropleuron (Valenciennes, 1840) in the northern part of Peninsular Malaysia, Tropical Life Sciences Research, 23, 59-65.

29. Nyanti L., Ling T.-Y. and Jongkar G., 2012 - Fish and crustacean communities and fish lengthweight relationship of Lutong River, Miri, Sarawak, Malaysia, World Journal of Fish and Marine Sciences, 4, 102-110.

30. Osborne J. W. and Amy O., 2004 - The power of outliers (and why researchers should ALWAYS check for them), Practical Assessment, Research and Evaluation, 9, 1-12.

31. Rahel F. J., 2007 - Biogeographic barriers, connectivity and homogenization of freshwater faunas: it's a small world after all, Freshwater Biology, 41, 51-54.

32. Ricker W. E., 1975 - Computation and Interpretation of Biological Statistics of Fish Populations, Bulletin of the Fisheries Research Board of Canada, Ottawa, Department of the Environment Fisheries and Marine Service, 191, 382.

33. Samat A., Izzati A. A., Nur-Hasyimah R., Shukor M. N. and Norhayati A., 2012 - Small streams ichthyofauna of Western Langkawi Island, Malaysia, Malaysian Applied Biology, 41, 51-54.

34. Santos M. N., Gaspar M. B., Vasconcelos P. and Monteiro C. C., 2002 - Weight-length relationship for 50 selected fish species of the Algarve coast, Fisheries Research, 59, 289-295.

35. Vié J.-C., Hilton-Taylor C. and Stuart S. N., 2008 - Wildlife in a Changing World - An Analysis of the 2008 IUCN Red List of Threatened Species, IUCN, Switzerland, 51, 180.

36. Yusof M. F., Siraj S. S. and Daud S. K., 2011 - Length-weight relationships of seven catfish species in Peninsular Malaysia, Journal of Fisheries and Aquatic Science, 6, 828-833.

37. Zakaria-Ismail M., 1991 - The fish fauna of the Ulu Endau River System, Johore, Malaysia, Malayan Nature Journal, 41, 403-411.

38. Zakaria M. Z., Jalal K. C. A. and Ambak M. A., 2000 - Length-weight relationship and relative condition factor of Sebarau, Hampala macropleidota (Van Hasselt) in Kenyir Lake, Malaysia, Pakistan Journal of Biological Sciences, 3, 721-724.

39. Zakeyudin M., Isa M. M., Rawi C. M., Shah A.-S. M. and Ahmad A.-H., 2012 - Assessment of suitability of Kerian River tributaries using length-weight relationship and relative condition factor of six freshwater fish species, Journal of Environment and Earth Science, 2, 52-61.

40. Zulkafli A. R., Amal M. N. A., Shohaimi S., Mustafa A., Ghani A. H., Hashim S., Hasfairi M. P., 2014 - Length-weight relationships of 20 fish species from Pahang River, Maran district, Pahang, Malaysia, Journal of Applied Ichthyology, 31, 409-410.

41. Zulkafli A. R., Amal M. N. A., Shohaimi S., Mustafa A., Ghani A. H., Hashim S. and Ayub S., 2015 - Length-weight relationships of 15 fish species from Tembeling River, Pahang, Malaysia, Journal of Applied Ichthyology, 32, 167-168.

42 Zulkafli A. R., Amal M. N. A., Shohaimi S., Mustafa A., Ghani A. H., Ayub S. and Hasfairi, P., 2016 - Length-weight relationships of 13 fish species from Pahang River, Temerloh District, Pahang, Malaysia, Journal of Applied Ichthyology, 32, 165-166. 